\title{
3 Modular and cultural factors in biological understanding: an experimental approach to the cognitive basis of science
}

\author{
Scott Atran \\ The experience that shaped the course of evolution offers no hint of the problems to be faced \\ in the sciences, and the ability to solve these problems could hardly have been a factor in \\ evolution. \\ - Noam Chomsky, Language and Problems of Knowledge, 1988, p.158
}

This essay explores the universal cognitive bases of biological taxonomy and taxonomic inference using cross-cultural experimental work with urbanized Americans and forest-dwelling Maya Indians. The claim is that there is a universal, essentialist appreciation of generic species as the causal foundation for the taxonomic arrangement of biodiversity, and for inference about the distribution of causallyrelated properties that underlie biodiversity. Generic species reflect characteristics of both the scientific genus and species. A principled distinction between genus and species is not pertinent to knowledge of local environments, nor to the history of science until after the Renaissance. Universal folk-biological taxonomy is domainspecific; that is, its structure does not spontaneously or invariably arise in other cognitive domains, such as the domains of substances, artefacts or persons. It is plausibly an innately-determined evolutionary adaptation to relevant and recurrent aspects of ancestral hominid environments, such as the need to recognize, locate, react to, and profit from many ambient species. Folk biology also plays a special role in cultural evolution in general, and particularly in the development of Western biological science. Although the theory of evolution may ultimately dispense with core concepts of folk biology, including species, taxonomy and teleology, in practice these may remain indispensable to doing scientific work.

\section{Introduction}

What follows is a discussion of three sets of experimental results that deal with various aspects of universal biological understanding among American and Maya children and adults. The first set of experiments shows that by the age of four-to-five years (the earliest age tested in this regard) urban American and Yukatek Maya 
children employ a concept of innate species potential, or underlying essence, as an inferential framework for understanding the affiliation of an organism to a biological species, and for projecting known and unknown biological properties to organisms in the face of uncertainty. The second set of experiments shows that the youngest Maya children do not have an anthropocentric understanding of the biological world.

Children do not initially need to reason about non-human living kinds by analogy to human kinds. The fact that American children show anthropocentric bias appears to owe more to a difference in cultural exposure to non-human biological kinds than to a basic causal understanding of folk biology per se. Together, the first two sets of experiments suggest that folk psychology can't be the initial source of folk biology. They also indicate that to master biological science, people must learn to inhibit activation of universal dispositions to view species essentialistically and to see humans as inherently different from other animals.

The third set of results show that the same taxonomic rank is cognitively preferred for biological induction in two diverse populations: people raised in the Mid-western USA and Itza' Maya of the Lowland Meso-american rainforest. This is the generic species - the level of oak and robin. These findings cannot be explained by domain-general models of similarity because such models cannot account for why both cultures prefer species-like groups in making inferences about the biological world, although Americans have relatively little actual knowledge or experience at this level. In fact, general relations of perceptual similarity and expectations derived from experience produce a 'basic level' of recognition and recall for many Americans that corresponds to the superordinate life-form level of folk-biological taxonomy the level of tree and bird. Still Americans prefer generic species for making inductions about the distribution of biological properties among organisms, and for predicting the nature of the biological world in the face of uncertainty. This supports the idea of the generic-species level as a partitioning of the ontological domains of plant and animal into mutually exclusive essences that are assumed (but not necessarily known) to have unique underlying causal natures.

The implication from these experiments is that folk biology may well represent an evolutionary design: universal taxonomic structures, centred on essencebased generic species, are arguably routine products of our 'habits of mind,' which may be in part naturally selected to grasp relevant and recurrent 'habits of the world.' The science of biology is built upon these domain-specific cognitive universals: folk 
biology sets initial cognitive constraints on the development of any possible macrobiological theory, including the initial development of evolutionary theory.

Nevertheless, the conditions of relevance under which science operates diverge from those pertinent to folk biology. For natural science, the motivating idea is to understand nature as it is 'in itself,' independently of the human observer (as far as possible). From this standpoint, the species-concept, like taxonomy and teleology, may arguably be allowed to survive in science as a regulative principle that enables the mind to readily establish stable contact with the surrounding environment, rather than as an epistemic concept that guides the search for truth.

\section{Four points of general correspondence between folk biology and scientific systematics}

In every human society, people think about plants and animals in the same special ways. These special ways of thinking, which can be described as 'folk biology, are basically different from the ways humans ordinarily think about other things in the world, such as stones, stars, tools or even people. The science of biology also treats plants and animals as special kinds of object, but applies this treatment to humans as well. Folk biology, which is present in all cultures, and the science of biology, whose origins are particular to Western cultural tradition, have corresponding notions of living kinds. Consider four corresponding ways in which ordinary folk and biologists think of plants and animals as special.

First, people in all cultures classify plants and animals into species-like groups that biologists generally recognize as populations of inter-breeding individuals adapted to an ecological niche. We call such groups - like redwood, rye, raccoon or robin - 'generic species' for reasons that will become evident. Generic species are usually as obvious to a modern scientist as to local folk. Historically, the genericspecies concept provided a pre-theoretical basis for scientific explanation of the organic world in that different theories - including evolutionary theory - have sought to account for the apparent constancy of 'common species' and the organic processes that centre on them (Wallace, 1901, p.1)

Second, there is a common-sense assumption that each generic species has an underlying causal nature, or essence, which is uniquely responsible for the typical appearance, behaviour and ecological preferences of the kind. People in diverse 
cultures consider this essence responsible for the organism's identity as a complex, self-preserving entity governed by dynamic internal processes that are lawful even when hidden. This hidden essence maintains the organism's integrity even as it causes the organism to grow, change form and reproduce. For example, a tadpole and frog are in a crucial sense the same animal although they look and behave very differently, and live in different places. Western philosophers, such as Aristotle and Locke, attempted to translate this common-sense notion of essence into some sort of metaphysical reality, but evolutionary biologists reject the notion of essence as such. Nevertheless, biologists have traditionally interpreted this conservation of identity under change as due to the fact that organisms have separate genotypes and phenotypes.

Third, in addition to the spontaneous division of local flora and fauna into essence-based species, such groups have 'from the remotest period in... history... been classed in groups under groups. This classification [of generic species into higherand lower-order groups] is not arbitrary like the grouping of stars in constellations' (Darwin 1872/1883, p.363). ${ }^{1}$ The structure of these hierarchically included groups, such as white oak / oak / tree or mountain robin / robin / bird, is referred to as 'folkbiological taxonomy.' Especially in the case of animals, these non-overlapping taxonomic structures can often be scientifically interpreted in terms of speciation (related species descended from a common ancestor by splitting off from a lineage).

Fourth, such taxonomies not only organize and summarize biological information; they also provide a powerful inductive framework for making systematic inferences about the likely distribution of organic and ecological properties among organisms. For example, given the presence of a disease in robins one is 'automatically' justified in thinking that the disease is more likely present among other bird species than among non-bird species. In scientific taxonomy, which belongs to the branch of biology known as systematics, this strategy receives its strongest expression in 'the fundamental principle of systematic induction' (Warburton, 1967; Bock, 1973). On this principle, given a property found among

\footnotetext{
${ }^{1}$ Thus, comparing constellations in the cosmologies of Ancient China, Greece and the Aztec Empire shows little commonality. By contrast, herbals like the Ancient Chinese ERH YA, Theophrastus's Peri Puton Istorias, and the Aztec Badianus Codex, share important features, such as the classification of generic species into tree and herb life forms (Atran, 1990, p.276).
} 
members of any two species, the best initial hypothesis is that the property is also present among all species that are included in the smallest higher-order taxon containing the original pair of species. For example, finding that the bacteria $E$ scheriehia coli share a hitherto unknown property with robins, a biologist would be justified in testing the hypothesis that all organisms share the property. This is because E. coli link up with robins only at the highest level of taxonomy, which includes all organisms. This or any general-purpose system of taxonomic inference for biological kinds is grounded in a universal belief that the world naturally divides into the limited causal varieties we commonly know as (generic) species.

\section{Universal folk-biological taxonomy}

In all societies that have been studied in depth, folk-biological groups, or taxa, are organized into ranks, which represent an embedding of distinct levels of reality. Most folk-biological systems have between three and six ranks (Berlin, 1992). Taxa of the same rank are mutually exclusive and tend to display similar linguistic, biological and psychological characteristics. Ranks and taxa, whether in folk-biological or scientific classification, are of different logical orders, and confounding them is a category mistake. Biological ranks are second-order classes of groups (e.g., species, family, kingdom) whose elements are first-order groups (e.g., lion, feline, animal). Folkbiological ranks vary little across cultures as a function of theories or belief systems. Ranks are universal but not the taxa they contain. Ranks are intended to represent fundamentally different levels of reality, not convenience. ${ }^{2}$

\subsection{Folk Kingdom}

The most general folk-biological rank is the folk kingdom. Examples are plant and

\footnotetext{
${ }^{2}$ Generalizations across taxa of the same rank thus differ in logical type from generalizations that apply to this or that taxon. Termite, pig and lemon tree are not related to one another by a simple class-inclusion under a common hierarchical node, but by dint of their common rank - in this case the level of generic species. A system of rank is not simply a hierarchy, as some suggest (Rosch, 1975; Premack, 1995; Carey, 1996). Hierarchy - that is, a structure of inclusive classes - is common to many cognitive domains, including the domain of artefacts. For example, chair often falls under furniture but not vehicle, and car falls under vehicle but not furniture. But there is no ranked system of artefacts: no inferential link, or inductive framework, spans both chair and car, or furniture and vehicle, by dint of a common rank, such as the artefact species or the artefact family.
} 
animal. Such taxa are not always explicitly named, and represent the most fundamental divisions of the biological world. These divisions correspond to the notion of 'ontological category' in philosophy (Donnellan, 1971) and psychology (Keil, 1979). From an early age, it appears, humans cannot help but conceive of any object they see in the world as either being or not being an animal (Inagaki and Hatano, 1994) and there is evidence for an early distinction between plants and nonliving things (Hatano and Inagaki, 1999).

\subsection{Life Form}

The next rank down is that of life form. Most taxa of lesser rank fall under one or another life form. Life-form taxa often have lexically unanalysable names (simple primary lexemes), such as tree and bird, although some life-form names are analysable, such as quadruped. Biologically, members of a life-form taxon are diverse. Psychologically, members of a life-form taxon share a small number of perceptual diagnostics: stem aspect, skin covering and so forth (Brown, 1984). Life-form taxa may represent adaptations to broad sets of ecological conditions, such as competition among single-stem plants for sunlight and tetrapod adaptation to life in the air (Hunn 1982; Atran, 1985). Classifying by life form may occur early on: two-year-olds distinguish familiar kinds of quadruped (e.g., dog and horse) from sea versus air animals (Mandler et al., 1991).

\subsection{Generic Species}

The core of any folk taxonomy is the generic-species level. Like life-form taxa, generic species are often named by simple lexemes, such as oak and robin.

Sometimes, generic species are labelled as binomial compounds, likes hummingbird. On other occasions, they may be optionally labelled as binomial composites, such as oak tree. In both cases the binomial makes the hierarchical relation apparent between generic and life form.

Generic species often correspond to scientific genera (e.g., oak) or species (e.g., dog), at least for the most phenomenally salient organisms, such as larger vertebrates and flowering plants. On occasion generic species can correspond to local fragments of biological families (e.g., vulture), orders (e.g., bat) and - especially with invertebrates - even higher-order biological taxa (Atran, 1987; Berlin, 1992). Generic species may also be the categories most easily recognized, most commonly named 
and most easily learned by children in small-scale societies (Stross, 1973). Indeed, ethnobiologists who otherwise differ in their views of folk taxonomy tend to agree that one level best captures discontinuities in nature and provides the fundamental constituents in all systems of folk-biological categorization, reasoning and use (Bartlett, 1940; Bulmer, 1974; Berlin, 1978; Hunn, 1982; Ellen, 1993).

Ethnobiologists, historians of systematics and field biologists mostly agree 'that species come to be tolerably well defined objects... in any one region and at any one time' (Darwin 1872/1883, p.137) and that such local species of the common man are the heart of any natural system of biological classification (Diamond and Bishop, 1999).

The term 'generic species' is used here, rather than 'folk genera / folk generic' or 'folk species / folk specieme,' for two reasons:

(1) Perceptually, a principled distinction between biological genus and species is not pertinent to most people around the world. For humans, the most phenomenally salient species (including most species of large vertebrates, trees, and evolutionarily isolated groups such as palms and cacti) belong to monospecific genera in any given locale. Closely related species of a polytypic genus are often hard to distinguish locally, and no readily perceptible morphological or ecological 'gap' can be discerned between them (Diver, 1940). ${ }^{3}$

(2) Historically, the distinction between genus and species did not appear until the influx of newly discovered species from around the world compelled European naturalists to sort and remember them within a worldwide system of genera built around (mainly European) species types (Atran, 1987). The original genus-concept was partially justified in terms of initially monotypic generic European species to which other species around the world might be attached (Tournefort, 1694).

People in all cultures spontaneously partition the ontological categories

\footnotetext{
${ }^{3}$ In a comparative study of Itza' Maya and rural Michigan college students, we found that the great majority of mammal taxa in both cultures correspond to scientific species, and most also correspond to monospecific genera: 30 of 40 (75\%) basic Michigan mammal terms denote biological species, of which $21(70 \%$, or $53 \%$ of the total) are monospecific genera; 36 of $42(86 \%)$ basic Itza' mammal terms denote biological species, of which $25(69 \%$, or $60 \%$ of the total) are monospecific genera (López et al., 1997). Similarly, a Guatemalan government inventory of the Itza' area of the Peten rainforest indicates that $69 \%$ (158 of 229) are monospecific (AHG/APESA 1992) the same percentage of monospecific tree genera (40 of 58) as in our study of the Chicago area (Medin et al.,
} 
animal and plant into generic species in a virtually exhaustive manner. 'Virtually exhaustive' means that when an organism is encountered that is not readily identifiable as belonging to a named generic species, it is still expected to belong to one. The organism is often assimilated to one of the named taxa it resembles, but sometimes it is assigned an 'empty' generic-species slot pending further scrutiny (e.g., 'such-and-such a plant is some [generic-species] kind of tree,' Berlin, 1999). This partitioning of ontological categories is part and parcel of the categories themselves: no plant or animal can fail in principle to belong uniquely to a generic species.

\subsection{Folk-specific}

Generic species may be further divided into folk-specifics. These taxa are usually labelled binomially, with secondary lexemes. Compound names, like white oak and mountain robin, make the hierarchical relation transparent between a generic species and its folk-specifics. Folk-specifics that have tradition of high cultural salience may be labelled with primary lexemes, such as winesap (a kind of apple tree) and tabby (a kind of cat). In general, whether and how a generic species is further differentiated depends on the cultural significance of the organisms involved. Occasionally, an important folk-specific taxon will be further sub-divided into contrasting folk-varietal taxa: for example, short-haired tabby versus long-haired tabby. Folk-varietals are usually labelled trinomially, with tertiary lexemes that make transparent their taxonomic relationship with superordinate folk-specifics and generic species, for example swamp white oak.

Thus, in addition to generic species, people everywhere tend to form groups both subordinate and superordinate to the level of preferred groups. Cultures across the world organize readily perceptible organisms into a system of hierarchical levels that are designed to represent the embedded structure of life around us, with the generic-species level being most informative. In some cultures people may develop 'theories' of life that are meant to cover all living kinds, such as Western theories of biology (Carey, 1985; Atran, 1990). But the very possibility of theorizing wouldn't exist without a universal construal of generic species to provide the trans-theoretical basis for scientific speculation about the biological world. 


\section{Folk biology doesn't come from folk psychology: Experiment 1}

One influential model of conceptual development in folk biology is Carey's (1985) notion that young children's understanding of living things is embedded in a folkpsychological, rather than folk-biological, explanatory framework, and that until age ten, it is based on their understanding of humans. Carey reports three major findings to bolster the claim that children's conceptions of the biological world are anthropocentric. First, projections from humans are stronger overall than projections from other living kinds. The other two findings are consequences of this difference in induction potential. The second result is that there are asymmetries in projection: inferences from human to mammals are stronger than from mammals to humans. Third, children violate projections according to similarity: inferences from humans to bugs are stronger than from bees to bugs. Together, these findings suggest that humans are the preferred base for children's inferences about the biological world.

This research has had a powerful impact on psychological theory and educational practice; but it suffers from a serious limitation. It has been conducted almost exclusively with individuals from North American, urban, technologicallyadvanced populations. In the few studies that go beyond this sample (e.g. studies by Inagaki and Hatano in Japan), the focus is still on urban, majority-culture children from advanced societies. Thus, it is not clear which aspects of children's naïve biology are likely to be universal and which depend critically on cultural conceptions and conditions of learning. We are also left with little insight into how to best design science curricula for non-majority, non-urban children. Human-centered reasoning patterns might reflect lack of knowledge about non-human living things rather than a radically different construal of the biological world. Consider results of the following experiment:

Participants: Participants were 98 Yukatek Maya-speaking children and 24 Yukatek Maya-speaking adults from rural villages in southcentral Quintana Roo, Mexico. 50 four-to-five year-olds and 48 six-to-seven year-olds were tested and included. Equal numbers of males and females were included in each group. By and large, the four-to-five year-olds were monolingual, the six-to-seven year-olds had begun learning Spanish, and almost all of the adults understood Spanish as a second language. All testing was done in Yukatek Maya.

Materials: Detailed colour drawings of objects were used to represent base 
and target categories. Four bases were used: Human, Dog, Peccary and Bee. Targets were divided into two sets. Each set included a representative of the categories Human (man, woman), Mammal (coatimundi, deer), Bird (eagle, chachalaca), Reptile (boa, turtle), Invertebrate (worm, fly), tree (Kanan, Gumbo Limbo), Stuff (stone, mud), Artefact (bicycle, pencil) and Sun (included in both sets). The children were tested on each set at different times, with both sets divided equally among girls and boys.

Procedure: Children were shown a picture of one of the bases and taught a new property about it. For example, the experimenter might show the dog picture, and say, 'Now, there's this stuff called andro. Andro is found inside some things. One thing that has andro inside is dogs. Now, I'm going to show you some pictures of other things, and I want you to tell me if you think they have andro inside like dogs do.' Participants were then shown each of the targets and asked: 'Does it have andro inside it, like the [base]?' Properties were unfamiliar internal substances of the form 'has X inside.' A different property was used for each base, and bases and targets were presented in random order for each participant.

\subsection{Results and discussion}

Each time a child projected a property from a base to a target it was scored 1, otherwise 0 . Responses did not differ reliably across any target pair (e.g., coatimundi vs. deer).

There was no overall preference in projections from humans. ${ }^{4}$ Projection from Human is least common across age groups, and inferences from Dog most common. Post hoc tests indicate that younger children project properties more readily than adults (older children do not differ from younger children or adults). Results show that inferences from Human are not stronger than those from any other base overall.

Another perspective on reasoning patterns is provided by analyses of how distinct patterns of generalization are for different bases. For this purpose we conducted trend analyses to look at projections as a function of similarity. We assume that birds are at distance 1 from mammals, reptiles at distance 2, insects at distance 3 and trees at distance 4 . The factors were AGE GROUP and SIMILARITY (distance)

\footnotetext{
${ }^{4}$ ANOVA: BASE (4) by GENDER (2) by AGE GROUP (3) revealed only a main effect of BASE: $\mathrm{F}(3,43)=16.2, p<.001$.
} 
and our interest was in the linear component. All age groups show clear similarity effects with dogs, peccaries and bees as bases. The generalization (similarity) gradients become sharper with age for dogs as a base. With humans as a base, fourto-five year-olds generalize broadly in an undifferentiated manner - they show no reliable effect of similarity. In contrast, adults show characteristically sharp gradients with humans as a base. The six-to-seven year-olds show a very weak similarity gradient. In short, the clearest developmental change is in determining the role of humans in the folk-taxonomic system (see Figure 1a).

- Figures 1a to 1d inserted about here -

There was no overall asymmetry in projection, as shown by conducting an ANOVA-test on the difference scores: The dependent variable for each subject was their base to target (e.g., Human to Mammal) score minus their target to base (e.g., Dog to Human) score. For inferences involving the bases Human and Dog, the data are inconsistent with Carey because only adults show the asymmetry favouring Human to Mammal over Dog to Human (see Figures 1a-1d).

There were no violations of similarity, again as shown by ANOVAs on difference scores: Calculated as mean projection from Human to target (e.g., Human to Mammal) minus mean projection from the target to a base in the same class as the target (e.g., Dog). Results are inconsistent with Carey because for all ages inferences from Dog to Mammal are better than inferences from Human to Mammal (see Figures 1a-1d).

\subsection{General discussion}

In sum, four-to-five year-old Yukatek Maya children do not show commitment to an anthropocentric understanding of the living world. This suggests that folk psychology is not a necessary or universal source for folk biology. Further support for this position comes from the Ross et al. (submitted) study of biological reasoning in three age groups (five-to-six, seven-to-eight, nine-to-ten years), among urban USA children and rural Native American children (Menominee). The projection task, like that used with Maya, was patterned after Carey (1985). Ross et al.'s sample of urban Boston children replicates major aspects of Carey's findings with Boston-area children. For example, Ross et al. find significant asymmetries between strength of projections from humans to categories in the same class (e.g., bear, raccoon) as the base (e.g., 
wolf), and the strength of projection from the non-human base (e.g., wolf) to humans for Boston five-to-eight year-olds. For young Menominee, however, Ross et al. find no evidence that projections from humans are reliably higher than projections from other bases. Neither do they find violations of similarity. Ross et al. argue that humans are the only animal that urban children know much about and so they generalize from them. Consistent with this view, Inagaki (1990) presents evidence that experience influences children's biological reasoning. She found that kindergarteners who raised goldfish were more likely than their counterparts who did not raise goldfish to reason about a novel aquatic animal (a frog) by analogy to goldfish rather than by analogy to humans.

Thus, Carey's results with Boston-area children cannot be generalized into a claim of biology's psychological origins. These findings are difficult to reconcile with Carey's strong claim of folk biology as the product of learned experience and radical conceptual change. If at least some basic aspects of the final or stable state of adult folk biology are pretty much the same across cultures, then there is no obvious sense to the notion that people in different cultural milieu acquire the same basic knowledge about folk biology through radical conceptual changes grounded in radically different learning environments. These results also undermine related claims that children acquire basic knowledge of the world as little scientists through successively novel theoretical formulations (Carey and Spelke, 1994), or that scientists come up with new theories because they manage to continue think like children (Gopnik et al., 1999a).

\section{Childhood conceptions of species essences: Experiment 2}

Young individuals have the potential to develop certain adult characteristics before those characteristics appear. The origins of these characteristics can be explained in two broadly different ways: nature and nurture. Some characteristics seem likely to develop from birth because they are essential to the species to which the individual belongs, such as a squirrel's ability to jump from tree to tree and hide acorns. Other characteristics are determined by the environment in which the individual is reared, such as a squirrel's fear or lack of fear of human beings.

Gelman and Wellman (1991) argue that young children predict categorytypical characteristics of individual animals based on the innate potential of the animal (i.e. the species of its birth-parent) rather than the environment in which it was 
raised (i.e. the species of its adoptive parent). Using an adoption study, they showed that four-year-old children judge that a baby cow raised by pigs will have the category-typical characteristics of cows (moos, straight tail) rather than pigs (oinks, curly tail). They interpret the results as showing that pre-schoolers believe that the innate potential or essence of species determines how an individual will develop, even in contrary environments.

This study is inconclusive with regard to children's assumptions about innate potential for two reasons. First, before the children in the study predicted the adult properties of the adopted baby, they were shown a drawing of the baby animal and told its species identity. Because the experimenters told the child that the baby and mother were of the same species, it does not address the question of how the children identify to which species the baby belongs in the first place (Johnson and Solomon, 1997). Second, the study explored only known facts about species and their associated properties. It did not examine whether or not children use the concept of species-essence or biological parentage as an inferential framework for interpreting and explaining hitherto unknown facts. It may be that a child has learned from experience, and as a matter of fact, that a calf is a cow because it was born to a cow. Still, the child may not know that being a member of a certain species causes a cow to be a cow (Carey, 1996). The current study was designed to test the extent to which children's assumptions about innate species-potential govern projection of both known and unknown properties, and to avoid the problems noted above (for details, see Atran et al., 2001).

Participants: Participants were 48 Yukatek Maya-speaking children and 24 Yukatek Maya-speaking adults. 24 four-to-five year-olds and 24 six-to-seven yearolds were tested and included. Equal numbers of males and females were included in each group. All testing was done in Yukatek Maya.

Procedure: In a forced choice task, children were asked whether an adult animal adopted at birth would resemble it's adoptive parent (e.g., cow) or birth-parent (e.g., pig) on four different individual traits: known behaviours (e.g. moo / oink), known physical features (e.g. straight / curly tail), unknown behaviours (e.g. looks for chachalacas / looks for pigeons), and unknown physical features (e.g. heart gets flatter / rounder when it is sleeping). Known traits were context-free, category-typical features that the children readily associated with species, whereas unknown traits were chosen to minimize any possibility of factual or pre-learned associations of 
traits with categories. Each unknown trait within a set was attributed to the birthparent for half the participants and to the adoptive parent for the other half. This assured that projection patterns of the unknown traits were not based on prior associations. The stories were accompanied by sketches of each parent. Sketches were designed to unambiguously represent a particular species of animal with minimum detail. In addition, sketches of known physical features (e.g. a sketch of a curly or straight tail), unknown physical features (e.g. flat vs. round heart) and relevant aspects of unknown behavioural contexts (e.g., closed vs. open eyes, mahogany vs. cedar tree) were shown to participants. These sketches in no way indicated the species to which the traits belonged. Participants indicated choice of birth or adoptive parent species by pointing to the relevant parent sketch.

The story was followed by two comprehension controls: a birth control (Who gave birth to the baby? Go ahead and point out the drawing of who gave birth to the baby) and a nurture control (Who did the baby grow up with?). If the child failed either control the adoption story was repeated and a second failure in comprehension resulted in exclusion of the child from the experiment. Children then were presented with the four experimental probes. For example: The cow mooed and the pig oinked. When the baby is all grown up will it moo like a cow or oink like a pig? For each set, the four probes (counter-balanced in order across children) were followed by a bias control in which the participant was asked: When the baby was growing up did it eat with animals that looked like X or animals that looked like Y?

A final probe involved a transformation story to explore the extent to which species essences are associated with inheritance versus vital internal properties as such (e.g., blood). Keil (1989, p.224), found that the younger children are undecided as to whether inheritance or internal properties are primarily responsible for an animal's species identity.

\subsection{Results and Discussion}

For each probe, participants were given a score of one if they chose the birth parent and zero if they chose the adoptive parent. A GENDER $x$ AGE GROUP x SET $x$ PROBE repeated-measures ANOVA indicated only a main effect of probe type, $F(5$, $62)=3.9, p<.01$. Each mean was tested against chance $(0.5)$ and results appear in Table 1. Overall, the results show a systematic and robust preference for attributions from the birth-parent. This preference was observed for all age groups and for known 
and unknown behaviour and physical properties. The trend is somewhat stronger in older children and adults and slightly stronger for known than unknown properties. Means for all probes were significantly different from chance, except the kind and blood for the youngest children. The kind probe was only marginally different from chance for the young children $(p=0.10)$, possibly because of the foreign character of the Mayanized Spanish word for kind, 'klaasej.' Results on the blood probe for the youngest children might suggest genuine indecision as to whether inheritance or vital internal functioning is primarily responsible for an animal's species identity. The low mean on the bias-control probe for all groups indicates that the method of the current experiment did not bias participant responses toward the birth-parent.

\subsection{General Discussion}

Results of this study indicate that Yukatek Maya children and adults assume that members of a species share an innate causal potential that largely determines category-typical behavioural and physical properties even in conflicting environments. Projection of properties to the birth-parent in the face of uncertainty and novelty implies that even young Maya children use the notion of underlying essence as an inferential framework for understanding the nature of biological species. By age seven, children have effectively attained adult competence in inferential use of the notion of innate species potential. These findings, together with Gelman and Wellman's (1991) earlier results for urban American children, suggest that such an essentialist bias in children is universal.

\section{Essence (Generic Species) vs. appearance (Basic Levels) in folk biology: Experiment 3}

In a justly celebrated set of experiments Rosch and her colleagues set out to test the validity of the notion of a psychologically preferred taxonomic level (Rosch et al., 1976). Using a broad array of converging measures they found that there is indeed a 'basic level' in category hierarchies of 'naturally occurring objects,' such as 'taxonomies' of artefacts as well as living kinds. For artefact and living kind hierarchies, the basic level is where: (1) many common features are listed for categories, (2) consistent motor programs are used for the interaction with or manipulation of category exemplars, (3) category members have similar enough shapes so that it is possible to recognize an average shape for objects of the category, 
(4) the category name is the first name to come to mind in the presence of an object (e.g., 'table' versus 'furniture' or 'kitchen table').

There is a problem, however: the basic level that Rosch et al. (1976) had hypothesized for artefacts was confirmed (e.g., hammer, guitar); however, the hypothesized basic level for living kinds (e.g., maple, trout), which Rosch initially assumed would accord with the generic-species level, was not. For example, instead of maple and trout, Rosch et al. found that tree and fish operated as basic-level categories for American college students. Thus, Rosch's basic level for living kinds generally corresponds to the life-form level, which is superordinate to the generic-species level (see Zubin and Köpcke, 1986, for German).

To explore the apparent discrepancy between preferred taxonomic levels in small-scale and industrialized societies, and the cognitive nature of ethnobiological ranks in general, we use inductive inference. Inference allows us to test for a psychologically preferred rank that maximizes the strength of any potential induction about biologically relevant information, and whether or not this preferred rank is the same across cultures. If a preferred level carries the most information about the world, then categories at that level should favour a wide range of inferences about what is common among members. (For detailed findings under a variety of lexical and property-projection conditions, see Atran et al., 1997; Coley et al., 1997.)

The prediction is that inferences to a preferred category (e.g., white oak to oak, tabby to cat) should be much stronger than inferences to a superordinate category (oak to tree, cat to mammal). Moreover, inferences to a subordinate category (swamp white oak to white oak, short-haired tabby to tabby) should not be much stronger than or different from inferences to a preferred category. What follows is a summary of results from one representative set of experiments in two very diverse populations: Mid-western Americans and Lowland Maya.

Participants: Participants were two sets of 12 adult Itza' Maya and two sets of 12 adult Mid-western Americans, with equal numbers of males and females. The Itza' are Maya Amerindians living in the Petén rainforest region of Guatemala. Until recently, men devoted their time to shifting agriculture, hunting and silviculture, whereas women concentrated on the myriad tasks of household maintenance. The Americans were self-identified as people raised in Michigan and recruited through an advertisement in a local newspaper.

Materials: Based on extensive fieldwork, we chose a set of Itza' folk- 
biological categories of the kingdom $(\mathrm{K})$, life-form $(\mathrm{L})$, generic-species $(\mathrm{G})$, folkspecific (S), and folk-varietal (V) ranks. We selected three plant life forms (che'= tree, $a k^{\prime}=$ vine, $p o k \sim c h e^{\prime}=$ herb/bush) and three animal life forms ( $b^{\prime} a$ 'al che' kuxi'mal = 'walking animal,' i.e., mammal, $c h$ 'iich' $=$ birds including bats, $k a ̈ y=$ fish). Three generic-species taxa were chosen from each life form; each generic species had a subordinate folk-specific, and each folk-specific had a salient varietal.

The properties chosen for animals were diseases related to the 'heart' (puksik'al), 'blood' (k'ik'el), and 'liver' (tamen). For plants, diseases related to the 'roots' (motz), 'sap' (itz) and 'leaf' (le'). Properties were chosen according to Itza' beliefs about the essential, underlying aspects of life's functioning. Properties used for inferences had the form, 'is susceptible to a disease of the $<$ root $>$ called $<X>$.' For each question, ' $\mathrm{X}$ ' was replaced with a phonologically appropriate nonsense name (e.g. 'eta') to minimize the task's repetitiveness.

Procedure: All participants responded to a list of over 50 questions in which they were told that all members of a category had a property (the premise) and were asked whether 'all,' 'few,' or 'no' members of a higher-level category (the conclusion category) also possessed that property. The premise category was at one of four levels, either life-form (e.g. $\mathrm{L}=$ bird), generic-species (e.g. $\mathrm{G}=$ vulture), folk-specific (e.g. $\mathrm{S}=$ black vulture), or varietal (e.g. $\mathrm{V}=$ red-headed black vulture). The conclusion category was drawn from a higher-level category, either kingdom (e.g. K = animal), life-form (L), generic-species $(\mathrm{G})$, or folk-specific $(\mathrm{S})$. Thus, there were ten possible combinations of premise and conclusion category levels: $\mathrm{L} \rightarrow \mathrm{K}, \mathrm{G} \rightarrow \mathrm{K}$, $\mathrm{G} \rightarrow \mathrm{L}, \mathrm{S} \rightarrow \mathrm{K}, \mathrm{S} \rightarrow \mathrm{L}, \mathrm{S} \rightarrow \mathrm{G}, \mathrm{V} \rightarrow \mathrm{K}, \mathrm{V} \rightarrow \mathrm{L}, \mathrm{V} \rightarrow \mathrm{G}$, and $\mathrm{V} \rightarrow \mathrm{S}$. For example, a folkspecific-to-life-form $(\mathrm{S} \rightarrow \mathrm{L})$ question might be, 'If all black vultures are susceptible to the blood disease called eta, are all other birds susceptible?' If a participant answered 'no,' the follow-up question would be 'Are some or a few other birds susceptible to disease eta, or no other birds at all?'

The corresponding life forms for the Americans were: mammal, bird, fish, tree, bush and flower. (On flower as an American life form see Dougherty, 1979.) The properties used in questions for the Michigan participants were 'have protein X,' 'have enzyme Y,' and 'are susceptible to disease Z.' These were chosen to be internal, biologically-based properties intrinsic to the kind in question, but abstract enough so that rather than answering what amounted to factual questions participants 
would be likely to make inductive inferences based on taxonomic category membership.

\subsection{Results and discussion}

Responses were scored in two ways. First we totalled the proportion of 'all or virtually all' responses for each kind of question (e.g., the proportion of times respondents agreed that if red oaks had a property, all or virtually all oaks would have the same property). Second, we calculated 'response scores' for each item, counting a response of 'all or virtually all' as 3, 'some or few' as 2 , and 'none or virtually none' as 1. A higher score reflected more confidence in the strength of an inference.

Figure 2a summarizes the results from all Itza' informants for all life forms and diseases, and shows the proportion of 'all' responses (black), 'few' responses (chequered), and 'none' responses (white). For example, given a premise of folkspecific (S) rank (e.g., red squirrel) and a conclusion category of generic-species (G) rank (e.g., squirrel), 49\% of responses indicated that 'all' squirrels, and not just 'some' or 'none,' would possess a property that red squirrels have. Figure $2 b$ summarizes results of Michigan response scores for all life forms and biological properties. Analyses used t-tests with significance levels adjusted to account for multiple comparisons.

Figures $2 \mathrm{a}$ and $2 \mathrm{~b}$ around here

Following the main diagonals of Figures $2 \mathrm{a}$ and $2 \mathrm{~b}$ refers to changing the levels of both the premise and conclusion categories while keeping their relative level the same (with the conclusion one level higher than the premise). Induction patterns along the main diagonal indicate a single inductively preferred level. Examining inferences from a given rank to the adjacent higher-order rank (i.e., $\mathrm{V} \rightarrow \mathrm{S}, \mathrm{S} \rightarrow \mathrm{G}$, $\mathrm{G} \rightarrow \mathrm{L}, \mathrm{L} \rightarrow \mathrm{K}$ ), we find a sharp decline in strength of inferences to taxa ranked higher than generic species, whereas $\mathrm{V} \rightarrow \mathrm{S}$ and $\mathrm{S} \rightarrow \mathrm{G}$ inferences are nearly equal and similarly strong. Notice that for 'all' responses, the overall Itza' and Michigan patterns are nearly identical.

Moving horizontally within each graph corresponds to holding the premise 
category constant and varying the level of the conclusion. ${ }^{5}$ We find the same pattern for 'all' responses for both Itza' and Americans as we did along the main diagonal. However, in the combined response scores ('all' + 'few') there is evidence of increased inductive strength for higher-order taxa among Americans versus Itza'. On this analysis, both Americans and Itza' show the largest break between inferences to generic species versus life forms. But only American subjects also show a consistent pattern of rating inferences to life-form taxa higher than to taxa at the level of folk kingdom: $\mathrm{G} \rightarrow \mathrm{K} v s . \mathrm{G} \rightarrow \mathrm{L}, \mathrm{S} \rightarrow \mathrm{K} v s . \mathrm{S} \rightarrow \mathrm{L}$, and $\mathrm{V} \rightarrow \mathrm{K} v s . \mathrm{V} \rightarrow \mathrm{L}$.

Finally, moving both horizontally and along the diagonal, regression analysis reveals a small but significant difference between Itza' inductions using conclusions at the generic-species versus folk-specific levels: $\mathrm{V} \rightarrow \mathrm{G}$ and $\mathrm{S} \rightarrow \mathrm{G}$ are modestly weaker than $\mathrm{V} \rightarrow \mathrm{S}$. For Michigan participants, the folk-specific level is not differentiated from the generic-species level. In fact, most of the difference between $\mathrm{V} \rightarrow \mathrm{G}$ and $\mathrm{V} \rightarrow \mathrm{S}$ inductions results from inference patterns for the Itza' tree life-form. There is evidence that Itza' confer preferential status upon trees at the folk-specific level (e.g. savannah nance tree). Itza' are forest-dwelling Maya with a long tradition of agroforestry that antedates the Spanish conquest (Atran and Ucan Ek', 1999; Atran et al., 1999).

\subsection{General discussion}

These results indicate that both the ecologically inexperienced Americans and the ecologically experienced Itza' prefer taxa of the generic-species rank in making biological inferences. These findings cannot be explained by appeals either to crossdomain notions of perceptual 'similarity' or to the structure of the world 'out there,' as most ethnobiologists contend (Hunn, 1976; Boster, 1991; Berlin, 1992). If inferential potential were a simple function of perceptual similarity then Americans should prefer life forms for induction (in line with Rosch et al.). Yet Americans prefer generic species as do Maya. Unlike Itza', however, Americans perceptually

\footnotetext{
${ }^{5}$ Moving vertically within each graph corresponds to changing the premise while holding the conclusion category constant. This allows us to test another domain-general model of category-based reasoning: The Similarity-Coverage Model (Osherson et al., 1990). In this model, the closer the premise category is to the conclusion category, the stronger induction should be. Our results show only weak evidence for this general reasoning heuristic, which fails to account for the various 'jumps' in inductive strength that indicate absolute privilege.
} 
discriminate life forms more readily than generic species (although one might expect that having less biodiversity in the American environment allows each species to stand out more from the rest). Despite the compelling needs established by lived experience, the Americans and Maya overwhelmingly, and in nearly equal measure, subordinate such influences to a preference for generic species.

The findings suggest that root categorization and reasoning processes in folk biology are due to domain-specific conceptual presumptions and not exclusively to domain-general, similarity-based (e.g., perceptual) heuristics. To be sure, language may signal expectation that little- or poorly-known generic species are more biologically informative than better known life forms for Americans (e.g., via common use of binomials, such as oak / red oak). Further experiments, however, show identical results in the absence of clear linguistic cues (e.g., oak / white oak / swamp white oak vs. dog / poodle / toy poodle - Coley et al., 1997).

Humans everywhere presume the biological world to be partitioned at the generic-species rank into non-overlapping kinds, each with its own unique causal essence, or inherent underlying nature, whose visible products may or may not be readily perceived. ${ }^{6}$ People anticipate that the biological information-value of these preferred kinds is maximal whether or not there is also visible indication of maximal co-variation of perceptual attributes. This does not mean that more general perceptual cues have no inferential value when applied to the folk-biological domain. On the contrary, the findings here point to a significant role for such cues in targeting basiclevel life-forms as secondary foci for inferential understanding in a cultural environment where biological awareness is poor, as among many Americans. Arguably, there is an evolutionary design to a cognitive division of labour between domain-general perceptual heuristics and domain-specific learning mechanisms: the one enabling flexible adaptation to variable conditions of experience, and the other invariably steering us to those abiding aspects of biological reality that are both causally recurrent and especially relevant to the emergence of human life and cognition (Atran, 1998).

\footnotetext{
${ }^{6}$ By contrast, a partitioning of artefacts (including those of organic origin, such as foods) is neither mutually exclusive nor inherent: some mugs may or may not be cups; an avocado may be a fruit or vegetable depending upon how it is served; a given object may be a bar stool or a waste bin depending on the social context or perceptual orientation of its user; and so on.
} 


\section{$7 \quad$ Science and common sense}

Much of the history of systematics has involved attempts to adapt locally relevant principles of folk biology to a more global setting, such as the taxonomic embedding of biodiversity, the primacy of species, and the teleo-essentialist causality that makes sense of taxonomic diversity and the life functions of species. This process has been far from uniform (e.g., initial rejection of plant but not animal life forms, recurrent but invariably failed attempts to define essential characters for species and other taxa, intermittent attempts to reduce teleological processes to mechanics, and so forth Atran, 1990).

Historical continuity between universal aspects of biological common sense and the science of biology should not be confounded with epistemic continuity or use of folk knowledge as a learning heuristic for scientific knowledge. Scientists have made fundamental ontological shifts away from folk understanding in the construal of species, taxonomy and underlying causality. For example, biological science today rejects fixed taxonomic ranks, the primary and essential nature of species, teleological causes 'for the sake' of species existence, and phenomenal evidence for the existence of taxa (e.g., trees do not constitute a scientifically valid superordinate plant group, but bacteria almost assuredly should).

Nevertheless, from the vantage of our own evolutionary history, it may be more important that our ordinary concepts be adaptive than true. Relative to ordinary human perceptions and awareness, evolutionary and molecular biology's concerns with vastly extended and minute dimensions of time and space has only marginal value. The ontological shift required by science may be so counter-intuitive and irrelevant to everyday life as to render inappropriate and maladaptive uses of scientific knowledge in dealing with ordinary concerns. Science can't wholly subsume or easily subvert folk-biological knowledge.

\subsection{Taxonomy}

Historically, taxonomy is conservative, but it can be revolutionized. Even venerable life forms, like tree, are no longer scientifically valid concepts because they have no genealogical unity (e.g., legumes are variously trees, vines, bushes, etc.). The same may be true of many long-standing taxa. Phylogenetic theorists question the 'reality' of zoological life forms, such as bird and reptile, and the whole taxonomic 
framework that made biology conceivable in the first place. Thus, if birds are descended from dinosaurs, and if crocodiles but not turtles are also directly related to dinosaurs, then: crocodiles and birds form a group that excludes turtles; or crocodiles, birds and turtles form separate groups; or all form one group. In any event, the traditional separation of bird and reptile is no longer tenable.

From Linnaeus to the present day, biological systematics has used explicit principles and organizing criteria that traditional folk might consider secondary or might not consider at all (e.g., the geometrical composition of a plant's flower and fruit structure, or the numerical breakdown of an animal's blood chemistry). Nevertheless, as with Linnaeus, the modern systematist initially depends implicitly, and crucially, on a traditional folk appreciation. As Bartlett (1936, p.5) noted with reference to the Maya of Peten (cf. Diamond, 1966, for zoology):

A botanist working in a new tropical area is... confronted with a multitude of species which are not only new to him, but which flower and fruit only at some other season than that of his visit, or perhaps so sporadically that he can hardly hope to find them fertile.... [C]onfronted with such a situation, the botanist will find that his difficulties vanish as if by magic if he undertakes to learn the flora as the natives know it, using their plant names, their criteria for identification (which frequently neglect the fruiting parts entirely), and their terms for habitats and types of land.

As Linnaeus needed the life form tree and its common species to actually do his work $(1738,1751)$, so did Darwin need the life form bird and its common species. From a strictly cosmic viewpoint, the title of his great work, On the Origins of Species, is ironic and misleading - much as if Copernicus had entitled his attack on the geocentric universe, On the Origins of Sunrise. Of course, in order to attain that cosmic understanding, Darwin could no more dispense with thinking about 'common species' than Copernicus could avoid thinking about the sunrise (Wallace 1889/1901, pp.1-2). In fact, not just species, but all levels of universal folk taxonomy served as landmarks for Darwin's awareness of the evolving pathways of diversity: from the folk-specifics and varietals whose variation humans had learned to manipulate, to intermediate-level families, and life-form classes, such as bird (Darwin 1872/1883, pp.353-354):

[In the Galapagos Islands] There are twenty-six land birds; of these twentyone or perhaps twenty-three are ranked a distinct species, and would 
commonly be assumed to have been here created; yet the close [family] affinity of most of these birds to American species is manifest in every character, in their habits, gestures, and tones of voice. So it is with other animals, and with a large proportion of plants.... Facts such as these, admit of no sort of explanation on the ordinary view of creation.

Use of taxonomic hierarchies in systematics today reveals a similar point. For example, by calculating whether or not the taxonomic diversity in one group varies over time as a function of the taxonomic diversity in another group, evidence can be garnered for or against the evolutionary interdependence of the two groups. Comparisons of the relative numbers of families of insects and flowering plants reveal the surprising fact that insects were just as taxonomically diverse before the emergence of flowering plants as after. Thus, evolutionary effects of plant evolution on the adaptive radiation of insects are probably less profound than previously thought (Labandeira and Sepkoski, 1993). The heuristic value of (scientifically elaborated) folk-based strategies for cosmic inquiry is compelling, despite scientific awareness that no 'true' distinctions exist between various taxonomic levels.

\subsection{The species concept}

In philosophy of biology, the current debate over the species-concept seems to centre on whether or not there is a single theoretically significant level of organization that covers all organisms (Kitcher, 1993; Sober, 1993). Accepting the primacy of evolutionary theory seems to rule out species-concepts that may be preferable on mainly pragmatic or operational grounds, such as historical primacy (Linnaean species), maximal covariance of many present and absent characters (pheneticists' basic taxonomic units), or minimally nested character-state distributions (speciation in pattern cladism).

Unfortunately, no one species-concept is presently able to simultaneously deal adequately with issues of inter-breeding (delimiting the boundaries of reproduction and gene flow), phylogenesis (fixing genealogical ascendance and descendance) and ecology (determining the geographical distribution of biodiversity ) - all of which seem fundamental to the causal patterning and development of life on earth (Hull, 1997). One response has been to call for pluralism, yielding as many species-concepts as may accord with various equal, different or combined considerations from psychology, history, logic, metaphysics or the several branches of biology 
(Ehreshefsky, 1992; Dupré, 1993). From the perspective of a bystander eyeing the multiplication of uses and abuses pluralism seems able to generate, such an alternative could well leave not only truth, but clarity as well, in the abyss.

Perhaps the species-concept, like teleology, should be allowed to survive in science more as a regulative principle that enables the mind to establish a regular communication with the ambient environment, than as an epistemic principle that guides the search for nomological truth. Once communication is established with the world, science may discover deeper channels, or more significant overlapping networks, of causality. The persistence of a species-concept would function to ensure only that that these diverse scientific explorations are never wholly disconnected or lost from one another, or from that aspect of phenomenal reality that will always remain as evident to a Maya Indian as to a modern scientist.

\subsection{Teleology}

Not only do taxonomic structure and species continue to agitate science - for better or worse - but so do the non-intentional and non-mechanical causal processes that people across the world assume to underlie the biological world. Vitalism is the folk belief that biological kinds - and their maintaining parts, properties and processes are teleological, and hence not reducible to the contingent relations that govern inert matter. Its cultural expression varies (cf. Hatano and Inagaki, 1994). Within any given culture people may have varying interpretations and degrees of attachment to this belief: some who are religiously inclined may think that a 'spiritual' essence determines biological causality; others of a more scientific temperament might hold that systems of laws which suffice for physics and chemistry do not necessarily suffice for biology. Many, if not most, working biologists (including cognitive scientists) implicitly retain at least a minimal commitment to vitalism: they acknowledge that physico-chemical laws should suffice for biology, but suppose that such laws are not adequate in their current form, and must be enriched by further laws whose predicates are different from those of inert physics and chemistry.

It is not evident how complete elimination of teleological expressions (concepts defined functionally) from biological theory can be pursued without forsaking a powerful and fruitful conceptual scheme for physiology, morphology, disease and evolution. In cognitive science, a belief that biological systems, such as the mind-brain, are not wholly reducible to electronic circuitry like computers, is a 
pervasive attitude that implicitly drives considerable polemic, but also much creative theorizing. As Kant (1790/1951) first intimated, even if vitalism represents a lingering folk belief that science ultimately seeks to discard, it remains an important and perhaps indispensable cognitive heuristic for regulating scientific inquiry. ${ }^{7}$

\section{Conclusion: emerging science in an evolutionary landscape}

The evolutionary argument for a naturally-selected cognitive disposition, such as folk biology, involves converging evidence from a number of venues: functional design (analogy), ethology (homology), universality, precocity of acquisition, independence from perceptual experience (poverty of stimulus), selective pathology (cerebral impairment), resistance to inhibition (hyperactivity), and cultural transmission. None of these criteria may be necessary, but the presence of all or some is compelling, if not conclusive.

\subsection{Functional Design}

All organisms must function to procure energy to survive, and they also must procure (genetic) information for recombination and reproduction (Eldredge, 1986). The first requirement is primarily satisfied by other species, and an indiscriminate use of any individual of the other species (e.g., energy-wise, it does not generally matter which chicken or apple you eat). The second requirement is usually only satisfied by genetic information unique to individual conspecifics (e.g., genetically, it matters who is chosen as a mate and who is considered kin). On the one hand, humans recognize other humans by individuating them with the aid of species-specific triggering algorithms that 'automatically' coordinate perceptual cues (e.g., facial recognition schemata, gaze) with conceptual assumptions (e.g., intentions) (Baron-Cohen, 1995). On the other hand, people do not spontaneously individuate the members of other species in this way, but as exemplars of the (generic) species that identifies them as

\footnotetext{
${ }^{7}$ Of course, most modern biologists no longer believe in strong vitalism, which holds that no reductive explanation is possible. Rather, many hold to a weaker form of vitalism, which disallows property or type reduction but allows reductive explanations of token biological processes. In other words, special-science processes must be realized in lower-level mechanisms on a token-for-token basis, although different token occurrences of the same higher-order type of process may have different varieties of lower-level realization. Strong vitalism is false, whereas weak vitalism might be true. My claim is only that there is a psychological bias to presume some sort of vitalism true.
} 
causally belonging to only one essential kind.

Natural selection basically accounts only for the appearance of complexly well-structured biological traits that are designed to perform important functional tasks of adaptive benefit to organisms. In general, naturally selected adaptations are structures functionally 'perfected for any given habit' (Darwin 1872/1883, p.140), having 'very much the appearance of design by an intelligent designer... on which the well-being and very existence of the organism depends' (Wallace 1889/1901, p.138). Plausibly, the universal appreciation of generic species as the causal foundation for the taxonomic arrangement of biodiversity, and for taxonomic inference about the distribution of causally-related properties that underlie biodiversity, is one such functional evolutionary adaptation. But a good story is not enough. ${ }^{8}$

\subsection{Ethology}

One hallmark of adaptation is a phylogenetic history that extends beyond the species in which the adaptation is perfected: for example, ducklings crouching in the presence of hawks, but not other kinds of birds, suggests dedicated mechanisms for something like species recognition. Some non-human species can clearly distinguish

\footnotetext{
${ }^{8}$ Although the adaptive relationship of structure to function is often manifest, as with the giraffe's neck or the rhinoceros's horns, often it is not. In such cases, evolutionary theorists adopt a strategy of 'reverse engineering.' Reverse engineering is what military analysts do when a weapon from an enemy or competitor in the arms market falls into their hands and they try to figure out exactly how it was put together and what it can do. Reverse engineering is easiest, of course, if the structure contains some signature of its function, like trying to figure out what a toaster does given the tell-tale sign of toasted bread-crumbs left inside. But in many cases recognizing the appropriate signs already requires some prior notion of what function the structure may have served. Thus, after a century and a half of debate, it is only now that scientists clearly favour the hypothesis that bipedality was primarily selected to enhance field of view. Comparative studies of humans with bipedal birds and dinosaurs, as well as experiments comparing energy expenditure and running speed in two-footed versus fourfooted running and walking, appear to exclude the competing hypotheses that bipedality evolved for running or energy conservation. For most higher-order human cognitive faculties, however, there may be little useful comparative evidence from elsewhere in the animal kingdom. This is because of their apparent structural novelty, poor representation in the fossil record (e.g., stone tools tell little of language or theory of mind) and lack of surviving intermediate forms. The moral is that reverse engineering can be helpful, and occasionally successful, but success is by no means guaranteed even in the richest of evidentiary contexts.
} 
several different animal or plant species (Lorenz, 1966; Cerella, 1979; Herrnstein, 1984). Vervet monkeys even have distinct alarm calls for different predator species or groups of species: snake, leopard and cheetah, hawk and eagle, and so forth (Hauser, 2000). Chimpanzees may have rudimentary hierarchical groupings of biological groups within groups (Brown and Boysen, in press). To be sure, the world itself is neither chaos nor flux: species are often locally self-structuring entities that are reproductively and ecologically isolated from other species through natural selection. But there is no a priori reason for the mind to always focus on categorizing and relating species qua species, unless doing so served some adaptive function (e.g., it makes little difference which tiger could eat a person or which mango a person could eat). And the adaptive functions of organisms rarely, if ever, evolve or operate in nature as all-purpose mechanisms.

\subsection{Universality}

Ever since the pioneering work of Berlin and his colleagues, evidence from ethnobiology and experimental psychology has been accumulating that all human societies have similar folk-biological structures (Berlin et al. 1973; Hunn, 1977, Hays, 1983; Brown, 1984; Atran, 1990, 1999; Berlin, 1992). These striking crosscultural similarities suggest that a small number of organizing principles universally define folk-biological systems. Basic aspects of folk-biological structure (e.g., taxonomic ranking, primacy of generic-species) seem to vary little across cultures as a function of theories or belief systems.

\subsection{Ease of acquisition}

Acquisition studies indicate a precocious emergence of essentialist folk-biological principles in early childhood that are not applied to other domains (Keil, 1995; Hatano and Inagaki, 1999; Atran et al., 2001).

\subsection{Independence from perceptual experience}

Experiments on inferential processing show that that humans do not make biological inductions primarily on the basis of perceptual experience or any general similaritybased metric, but on the basis of imperceptible causal expectations of a peculiar, essentialist nature (Atran et al., 1997; Coley et al., 1997). 


\subsection{Pathology}

Cerebral impairments (Williams syndrome, brain lesions caused by certain types of herpes virus, etc.) suggest selective retention or loss of folk-biological taxonomies or of particular taxonomic ranks. Neuropsychological studies have reported a pathological performance in recognition at the life-form and generic-species levels (e.g., recognizing an item as an animal but not as a bird or robin), and dissociation at the life-form level (e.g., not recognizing items as trees). Existing studies, however, do not say anything about the generic-species rank as the preferred level of representation for reasoning, perhaps because of methodology (linked to averaging over items and failure to include sets of generic species) (Warrington and Shallice, 1984; Sartori and Job, 1988; Job and Surian, 1998).

\subsection{Inhibition and hyperactivity}

One characteristic of an evolved cognitive disposition is evident difficulty in inhibiting its operation (Hauser, 2000). Consider beliefs in biological essences. Such beliefs greatly help people explore the world by prodding them to look for regularities and to seek explanations of variation in terms of underlying patterns. This strategy may help bring order to ordinary circumstances, including those relevant to human survival. But in other circumstances, such as wanting to know what is correct or true for the cosmos at large, such intuitively ingrained concepts and beliefs may hinder more than help. For example, the essentialist bias to understand variation in terms of deviance is undoubtedly a hindrance to evolutionary thinking. In some everyday matters, the tendency to essentialise or explain variation in terms of deviation from some essential ideal or norm (e.g., people as mental or biological 'deviants') can be an effortlessly 'natural' but wrong way to think.

Because intuitive notions come to us so naturally they may be difficult to unlearn and transcend. Even students and philosophers of biology often find it difficult to abandon common-sense notions of species as classes, essences or natural kinds in favour of the concept of species as a logical individual - a genealogical branch whose endpoints are somewhat arbitrarily defined in the phyletic tree and whose status does not differ in principle for that of other smaller (variety) and larger (genus) branches. Similarly, racism - the projection of biological essences onto social groups - seems to be a cognitively facile and culturally universal tendency (Hirschfeld, 1996). Although science teaches that race is biologically incoherent, 
racial thinking is as notoriously difficult to suppress as it is easy to incite.

\subsection{Cultural transmission}

Human cultures favour the rapid selection and stable distribution of those ideas that: (1) readily help to solve relevant and recurrent environmental problems, (2) are easily memorized and processed by the human brain, and (3) facilitate the retention and understanding of ideas that are more variable (e.g., religion) or difficult to learn (e.g., science) but contingently useful or important. Folk-biological taxonomy readily aids humans everywhere in orienting themselves and surviving in the natural world. Its content tends to be stable within cultures (high inter-informant agreement, substantial historical continuity) and its structure isomorphic across cultures (see Boster, 1991; López et al., 1997). Folk-biological taxonomy also serves as a principled basis for transmission and acquisition of more variable and extended forms of cultural knowledge.

Consider, for example, the spontaneous emergence of totemism - the correspondence of social groups with generic species - at different times and in different parts of the world. Why, as Lévi-Strauss (1963) aptly noted, are totems so 'good to think'? In part, totemism uses representations of generic species to represent groups of people; however, this pervasive meta-representational inclination arguably owes its recurrence to its ability to ride piggyback on folk-biological taxonomy. Generic species and groups of generic species are inherently well-structured, attention-arresting, memorable and readily transmissible across minds. As a result, they readily provide effective pegs on which to attach knowledge and behaviour of less intrinsically well-determined social groups. In this way totemic groups can also become memorable, attention-arresting and transmissible across minds. These are the conditions for any idea to become culturally viable. (See Sperber, 1996, for a general view of culture along the lines of an 'epidemiology of representations'.) A significant feature of totemism that enhances both memorability and its capacity to grab attention is that it violates the general behaviour of biological species: members of a totem, unlike members of a generic species, generally do not inter-breed, but only mate with members of other totems in order to create a system of social exchange. Notice that this violation of core knowledge is far from arbitrary. In fact, it is such a pointed violation of human beings' intuitive ontology that it readily mobilizes most of the assumptions people ordinarily make about biology in order to better help build 
societies around the world (Atran and Sperber, 1991).

In sum, folk-biological concepts are special players in cultural evolution, whose native stability derivatively attaches to more variable and difficult-to-learn representational forms, thus enhancing the latter's prospects for regularity and recurrence in transmission within and across cultures. This includes knowledge that cumulatively enriches (e.g., to produce folk expertise), overrides (e.g., to produce religious belief) or otherwise transcends (e.g., to produce science) the common-sense ontology prescribed by folk biology. The theory of evolution may ultimately dispense with core concepts of folk biology, including species, taxonomy and teleology, but in practice these may remain indispensable to scientific work. Theory-driven scientific knowledge also cannot banish folk knowledge from everyday life.

To say an evolved mental structure is 'innate' is not to say that every important aspect of its phenotypic expression is 'genetically determined.' The particular organisms observed, actual exemplars targeted, and specific inferences made can vary significantly from person to person. Much as mountain rain will converge to the same mountain-valley river basin no matter where the rain falls, so each person's knowledge will converge on the same cognitive 'drainage basin' (Waddington, 1959; Sperber, 1996). This is because: (1) inputs naturally cluster in causally redundant ways inasmuch as that's the way the world is (e.g., where there are wings there are beaks or bills, where there are predators there are prey, where there are fruit-eating birds there are fruit-bearing trees, etc.); and (2) dedicated mental modules selectively target these inputs for processing by domain-specific inferential structures (e.g., to produce natural taxonomies). In this way, the mind is able to take fragmentary instances of a person's experience (relative to the richness and complexity of the whole data set) and spontaneously predict (project, generalize) the extension of those scattered cases to an indefinitely large class of intricately related cases (of larger relevance to our species and cultures). Thus, many different people, observing many different exemplars of dog under varying conditions of exposure to those exemplars, all still generate more or less the same general concept of $d o g$. Within this evolutionary landscape of medium-sized objects that are snapshot in a single lifespan of geological time, biologically-poised mental structures channel cognitive development but do not determine it. Cultural life, including science, can selectively target and modify parts of this landscape but cannot simply ignore it or completely replace it. 


\section{Acknowledgements}

The comparative studies reported here were co-directed with Douglas Medin and funded by NSF (SBR 97-07761) and the Russell Sage Foundation. 